\title{
Study on Vehicle Fuel Consumption and Emission Characteristics Based on Floating Vehicle Data of Beijing
}

\author{
Yanlin Wang ${ }^{1, a}$, Chenkun Yin ${ }^{1, b}$ \\ 1 School of Electronics and Information Engineering,Beijing Jiaotong University, Beijing 100044 \\ aemail, ${ }^{\text {bemail, }}$
}

Keywords: Floating Vehicle Data, Vehicle Fuel Consumption, PM2.5, Beijing

\begin{abstract}
In this paper, we analyzed the collected measured data of floating vehicles with mobile collection equipment in the driving of Beijing. The results show that the fuel consumption of the floating vehicle increases with the increase of the vehicle speed when the vehicle is traveling at a constant speed, and there is a significant difference between the two kinds of speed, which is consistent with the curve of the VT-Model. The fuel consumption per $100 \mathrm{~km}$ decreases first and then increases with the increase of the vehicle speed. There is a quadratic fit between the two kinds of speed, which is consistent with the curve of the Power-based Emission Model. PM2.5 is significantly related to instantaneous fuel consumption, but no typical function can be fitted, indicating that vehicle emission is not a decisive factor for PM2.5; the return value of pre-oxygen sensor decreases first with the increase of vehicle speed and there is a quadratic function fitting relationship between them, which also validates the concept of economic speed.
\end{abstract}

\section{Introduction}

Nowadays, automobile has become the most frequently used means of transportation, but the level of automobile technology in our country is relatively backward, the fuel consumption of bicycle is obviously higher than that of abroad. This will bring great pressure to the energy use of our country [1] [2]. Therefore, in the current new energy technology has not been able to use a wide range of circumstances and the study of vehicle fuel consumption is the key to solve this problem [3]. At present, the domestic and foreign research on vehicle fuel consumption model from the modeling method can be divided into real test regression model (also known as the multiple regression model), theoretical fuel consumption model and bench test fuel consumption model [4]. This paper analyzes the relationship between the fuel consumption characteristics and other parameters of the floating vehicle in the actual road driving process, which belongs to a multiple regression modeling method.

In addition to energy pressure, the popularity of the use of vehicles on our environment has also caused serious impact on urban road vehicle exhaust emissions has become China's urban air pollution, the most important source [5]. In particular, PM2.5 pollution caused widespread concern in today's society, motor vehicle emissions to its contribution rate reached $13.17 \%$, second only to coal and other industrial production processes and ground dust [6]. Therefore, it is a hotspot in the research to reduce the environmental pollution caused by vehicle emissions by analyzing the influence parameters related to the pollution discharge quantity and then implementing various traffic management and control measures. At present, there are five main methods to determine emission factors of motor vehicle bike in foreign countries: bench test method, tunnel experiment method, road vehicle test method, road remote sensing test method and emission model method, among which the emission model method is divided into macroscopic model and Based on motor vehicle driving characteristics of the model (micro-model) [7]. The VT-Micro model validated by the measured data in this paper belongs to the microscopic model in the emission model.

Although the existing fuel consumption model and emission model have more mature theoretical results, but there are many parameters of the model, the use of off-site is not strong enough to support the measured data and other defects. In order to solve these problems, this paper excavates and studies the correlation characteristics of fuel consumption and based on the collected large-scale original data, analyzes the relationship among the parameters and establishes the 
corresponding parameter model; the model proves the practical significance of the previous theoretical model.

\section{Data Description}

The Introduction of Floating Car Raw Data. Floating Car Data (FCD) is an important road traffic data source. As the taxi has a high probability of travel, long running time, wide range of traffic, the proportion of the higher proportion of traffic flow, so our country is generally a taxi as a float vehicle data collection medium.

The data used in this paper is from December 31, 2013, Beijing, about 2400 vehicles equipped with mobile phone equipment, taxi collected data. The device records the speed, fuel consumption, emissions, and position of the car every second. The total size of the original data after decompression is about 400G. There are 17 parameters recorded in the original data, but 10 parameters are analyzed in this paper, and their explanations are shown in Table 1.

Table 1. Main raw parameter variable descriptions

\begin{tabular}{c|c|c}
\hline \hline Variable Name & Field Description & Type Data \\
\hline GPS_LAT & GPS latitude & float \\
\hline GPS_LONG & GPS longitude & float \\
\hline GPS_TIME & GPS time & date \\
\hline IMEI & Vehicle Identification Code & int \\
\hline after_oxy & After the oxygen sensor voltage $(\mathrm{V})$ & int \\
\hline db_speed & Dashboard speed $(10 \mathrm{~km} / \mathrm{h})$ & int \\
\hline front_oxy & Pre- oxygen sensor voltage value & int \\
\hline instant_fuel & Instantaneous fuel consumption & int \\
\hline speed & Rotating speed $(1000 \mathrm{R} / \mathrm{min})$ & int \\
\hline torque & Torque $(20 \mathrm{~kg} * \mathrm{~m})$ & int \\
\hline \hline
\end{tabular}

The vehicle identification code is the only corresponding with each vehicle configuration of the mobile device; instantaneous fuel consumption, also known as the unit fuel consumption, is the basic indicator of vehicle fuel consumption; front oxygen sensor reflects the engine exhaust emissions in the oxygen content.

In this paper, acceleration, fuel consumption per $100 \mathrm{~km}$ and PM2.5 are introduced in the analysis, and their explanations are shown in Table 2.

Table 2. Introduced parameter variable descriptions

\begin{tabular}{c|c|c}
\hline \hline Variable Name & Field Description & Data Type \\
\hline PM & $\begin{array}{c}\text { The corresponding PM2.5 value per } \\
\text { hour } \\
\text { Q }\end{array}$ & float \\
\hline a & $\begin{array}{c}\text { The acceleration is calculated } \\
\mathrm{km} \text { fuel consumption }\end{array}$ & float \\
& according to the formula (3) $\left(\mathrm{m} / \mathrm{s}^{2}\right)$ & \\
\hline
\end{tabular}

The value of PM is calculated according to the recorded value published by the US Embassy in China. The value of PM is updated every hour. The maximum value of PM2.5 and the minimum value of PM2.5 are recorded in this hour. The PM value is calculated according to the formula (1).

$$
P M=\frac{p_{\max }+p_{\min }}{2}
$$

$100 \mathrm{~km}$ fuel consumption is a car at a uniform speed when the fuel consumption is a reflection of 
the basic indicators of vehicle fuel consumption, according to the formula (2) calculated. Where Q expresses the fuel consumption of the constant speed vehicle $100 \mathrm{~km}$, instant_fuel indicates the instantaneous fuel consumption of the vehicle, and db_speed indicates the current traveling speed of the vehicle.

$$
Q=\frac{3600 \times \text { instant_fuel }}{d b_{\text {_ }} \text { speed }}
$$

Acceleration is calculated according to the formula (3), where Vt1 is the corresponding dashboard speed at time t1, Vt2 is the corresponding dashboard speed at time t2, and a is the acceleration at time $\mathrm{t} 1$.

$$
a=\frac{V_{t_{2}}-V_{t_{1}}}{t_{2}-t_{1}}
$$

Data Preprocessing. This article uses the processing method is to use the first batch of all the text files into a text file, and then import the text file to the database through the establishment of SQL Server. But because the original data is collected by the second, the calculation of particle size is too small; In order to improve efficiency, this paper takes a data from the original data every 20s to extract the data to compress the data. Then use the SQL command to clean the compressed data, to get rid of duplicate data, independent of the property data and status error data; then according to the GPS positioning range to remove the data outside the Beijing area. In the process of missing data, missing data is neglected because of the small proportion of missing data and the sufficient sample size of the measured data.

After the data is cleaned, the singular value analysis of the parameters is followed. Since the three most important parameters in this paper are front_oxy, db_speed, and instant_fuel, the distribution of the values of these three variables can be fitted with the density function curve as shown in equation (4). Therefore, these three variables are processed according to the $3 \sigma$ principle to improve the data quality.

$$
\varphi(\mathrm{x})=\frac{1}{\sqrt{2 \pi}} e^{-\frac{(\mathrm{x}-\mu)^{2}}{2 \sigma^{2}}}(x \in R)
$$

Finally, through the SQL instruction to calculate the PM, Q and a these three new variables to complete the construction of the entire database, the database is 8602122 effective record.

\section{Analysis of Fuel Consumption Characteristics}

In the process of modeling the parameters, we must first analyze the correlation of the variables; after modeling, we should also test the consistency of the model. In this paper, we use the Pearson correlation coefficient, Spearman correlation coefficient and decision coefficient [8].

The Relationship Analysis between Instantaneous Fuel Consumption and Speed. Car driving state can be divided into idling, uniform speed and acceleration of these three, different driving conditions will lead to different fuel consumption. [9] It presents a VT-Micro model curve $(\mathrm{a}=0)$, the formula (5) gives the mathematical expression of the model.

$$
M O E_{e}=\exp \left(\sum_{i=0}^{3} \sum_{j=0}^{3}\left(K_{i, j}^{e} \times v^{i} \times a^{j}\right)\right)
$$

Where MOEe is the instantaneous fuel consumption of the vehicle, $\mathrm{v}$ and a represent the speed and acceleration of the vehicle, and Kei, $\mathrm{j}$ is the coefficient of the model. It is clear that the model is exponential.

The correlation between instant_fuel and db_speed was analyzed by Pearman correlation coefficient. It was found that there was a significant correlation between the two parameters in the case of constant speed driving $(\mathrm{a}=0 \mathrm{~m} / \mathrm{s} 2)$.

The regression formula of instant_fuel and db_speed can be obtained by regression calculation of SPSS software, as shown in formula (6).

$$
\text { instant_fuel }=120.175 \times e^{0.022 \times d b \_s p e e d}
$$


Figure 1 shows the instant fuel and db_speed the fitting curve.

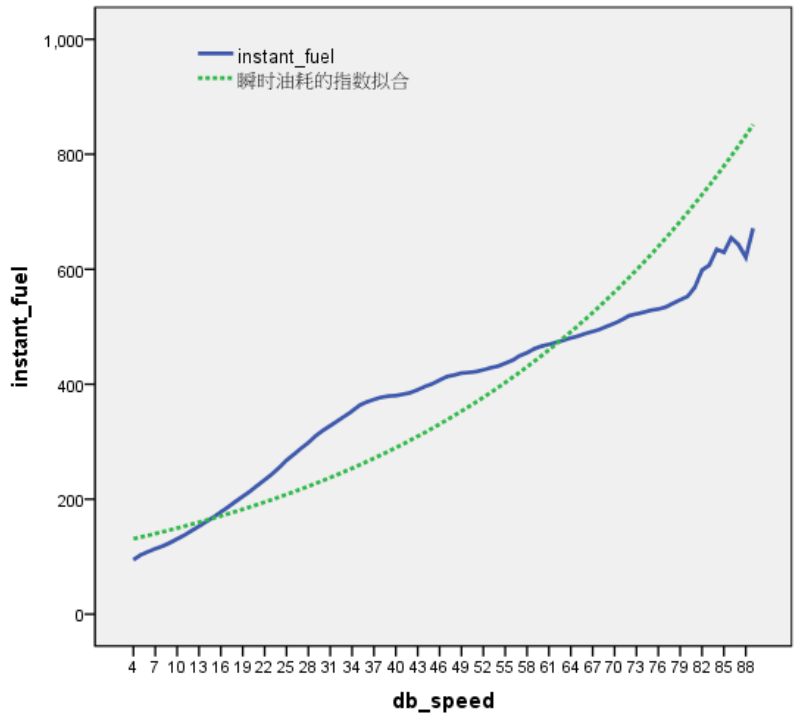

Figure 1: The exponential fit between instantaneous fuel consumption and speed

It can be seen from Figure 1 with the constant speed of the vehicle speed increases, the instantaneous fuel consumption was an increasing trend, which in reality the higher the speed of motor vehicles, the engine work done more, the actual fuel consumption rate is relatively high consistent with the fact. Moreover, the curve is consistent with the curve of VT-Micro model $(\mathrm{a}=0)$, both of which are exponential function form, which also verifies the practicability of the model.

Analysis of the Relationship between $100 \mathrm{Km}$ Fuel Consumption and Vehicle Speed at Constant Speed. Studies have shown that in the actual driving process of vehicles, there is a concept of economic speed. When Q is used as a measure of fuel consumption, instant_fuel is relatively high at higher average speeds, but its Q value is lower than the $\mathrm{Q}$ at lower average speeds due to the relatively long distance traveled [10] . In order to verify this, the Spearman correlation coefficient between $\mathrm{Q}$ and $\mathrm{db}$ _speed is calculated, and the result is shown as 0.785 , indicating a significant correlation between the two parameters. Then the quadratic curve fitting formula between the two parameters is obtained by regression calculation of SPSS software, as shown in formula (7):

$$
\begin{gathered}
Q=16.224-0.251 \times d b_{-} \text {speed } \\
+0.002 \times d b_{-} \text {speed }^{2}
\end{gathered}
$$

Figure 2 shows the fuel consumption of the same speed $100 \mathrm{~km}$ quadratic curve fitting curve.

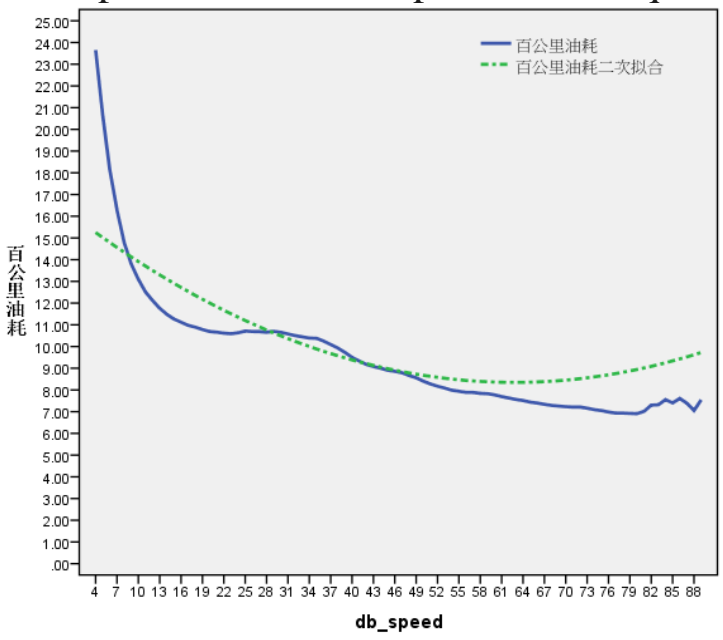

Figure 2: The quadratic fitting of $100 \mathrm{~km}$ fuel consumption and vehicle speed

This curve also verifies that the $\mathrm{Q}$ value does not increase with increasing vehicle speed. This is because when the car is running at high speed, it is moving higher gears, making the engine speed are not necessarily high, with high speed when the car's driving time is shortened, so this happens. 
This curve is consistent with the curve of Power-based Emission Model [11] proposed by the University of Hong Kong, which is a form of quadratic function. It also verifies the practicability of this model with measured data.

\section{Vehicle Emission Characteristics Analysis}

Analysis of the Relationship between PM2.5 and Instantaneous Fuel Consumption. Calculating the Spearman correlation coefficient between PM and other variables reveals that only the value of $\mathrm{db}$ speed is significantly related to it, whereas the value of the post-oxygen sensor, which is significantly related to it, is not related to it. Figure 3 shows the timing diagram for PM and db_speed.

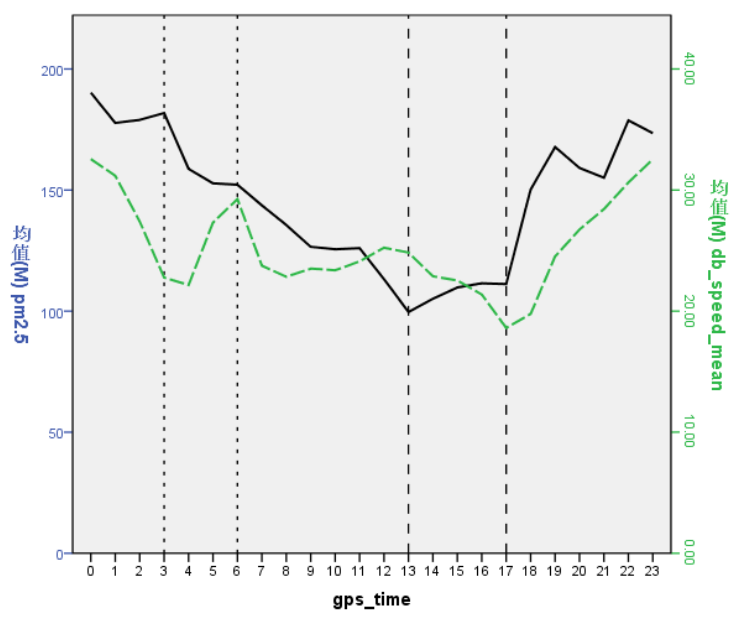

Figure 3: PM and db speed timing diagram

From Figure 3, it can be seen that the trend of the two variables is basically consistent; and PM2.5 peak with db_speed peak compared to the existence of 3 - 4 hours delay, because the vehicle emissions on the atmosphere there lag, with the reality of the situation.

However, after analysis, PM2.5 although with the instantaneous fuel consumption is significantly related, but there is no suitable model to achieve. It is also verified that the fuel consumption of the vehicle in the literature [6] is a factor of PM2.5, but it is not the influencing factor of the decisive effect.

Analysis of the Relationship between Pre-Oxygen Sensor and Instant Fuel Consumption. The voltage value of the front oxygen sensor reflects the oxygen content in the vehicle exhaust gas, from the value of the size of the driving process can analyze the adequacy of fuel use. By Pearson correlation analysis, it is found that the value of pre-oxygen sensor is significantly correlated with db_speed in the case of segmentation. So the front_oxg segmentation fitting, formula (8) is the fitting formula; which variable db_speed with $\mathrm{x}$ that variable front_oxg with $\mathrm{y}$ said.

$$
y=\left\{\begin{array}{l}
84.221+0.922 \times x-0.029 \times x^{2}, 1<x \leq 35 \\
135.174-2.19 \times x+0.019 \times x^{2}, 35<x \leq 75 \\
164.507-2.509 \times x+0.018 \times x^{2}, 75<x \leq 100
\end{array}\right.
$$

Figure 4 shows the fitting curve of the pre-oxygen sensor voltage and instantaneous fuel consumption. 


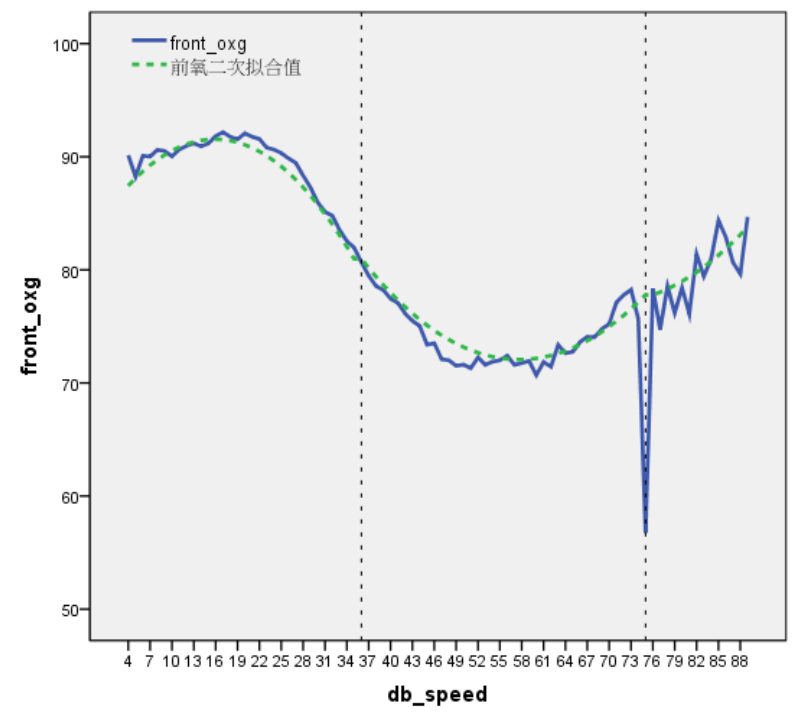

Figure 4: Piecewise quadratic fitting curves for pre-oxygen sensors and transient fuel consumption

It can be seen clearly from Fig. 4 that the value of the pre-oxygen sensor does not increase as the velocity increases, but reaches the maximum value when the velocity rises to about $22 \mathrm{~km} / \mathrm{h}$, then the inverse-growth phenomenon occurs, / $\mathrm{H}$ to reach the minimum, indicating that in this speed range, the maximum efficiency of the use of the engine can be more fully use of fuel, oxygen consumption is relatively large; and $22-60 \mathrm{~km} / \mathrm{h}$ this range is The so-called economic speed [12].

\section{Conclusion}

Based on the measured data collected from Beijing floating car, the data are pretreated, then the data are analyzed by SPSS software. Curve regression method is used to fit the curve between the instantaneous fuel consumption with the vehicle speed, the curve between $\mathrm{Km}$ fuel consumption with the speed and the curve between pre-oxygen sensor with the speed.

The velocity-instantaneous fuel consumption curve agrees well with the widely used VT-Micro model. The velocity-constant $100 \mathrm{~km}$ fuel consumption curve is in good agreement with the power-based Emission Mode. It overcomes the defects such as the too much theoretical model parameters, off-site use is not strong and there is not enough data to support the results.

PM2.5 is significantly correlated with instantaneous fuel consumption, but there is no appropriate curve fitting curve to express. This verifies that the fuel consumption of a motor vehicle is a factor that affects PM2.5, but is not a determinant factor.

The value of the pre-oxygen sensor has the quadratic function fitting relationship with the vehicle speed. There is an economic speed. When the vehicle runs within this speed range, the efficiency of the engine is the highest and the fuel can be fully utilized.

Through the above analysis of the relationship between fuel consumption and emissions, it can provide a reliable reference for urban road planning and design, and provide realistic basis for the design and improvement of automobile manufacturing industry, which has certain practical significance.

\section{References}

[1] Erickson J J, Greene D L, Sabadell A J. an analysis of transportation energy conservation projects in developing countries[J]. Transportation. 1988, 15(3)

[2] Yan Xiang. Entertainment of Environmental Protection Advanced City in Green Olympics: Implications for US Fuel Consumption Changes [J]. World Journal of Standardization and Quality Management2005 (11): 55-56.

[3] Zhang Maihua, Zhang Yafen. Analysis on the Current Situation and Developing Trend of 
China's Oil Dependence[J]. Liaoning Economy. 2006 (03): 30-31.

[4] Kui Hailin, Wang Jinsong, Wang Yunpeng, et al. An automobile fuel consumption model based on urban road condition [J] .Jilin University (Engineering and Technology Edition). 2009 (05): 1146-1150.

[5] Laws to Protect against Pollution- - An interview with Pan Yue, vice minister, the State Environmental Protection Administration(SEPA)[J]. China Economist. 2007(03): 5-9.

[6] Wang Zhijuan, Han Lihui, Chen Xufeng, et al. Characteristics and Sources of PM2.5 Pollution in Beijing [J] .Safety and Environment 2012 (05): 122-126.

[7] Huo Hong, He Kebin, Wang Qidong. Measurement of Motor Vehicle Pollution Emission Model[J] . Environmental Pollution and Control. 2006 (07): 526-530.

[8] Zhang Liangjun, Yang Tan, Xiao Gang, etc. MATLAB data analysis and mining combat [M]. Machinery Industry Press, 2015

[9] Rakha H, Ahn K, Trani A. Development of VT-Micro model for estimating hot stabilized light duty vehicle and truck emissions[J]. Transportation Research Part D Transport \& Environment. 2004, 9(1): 49-74.

[10] Tu Zhao. Study on Road Fuel Consumption Algorithm of Light Vehicle Based on Floating Vehicle Data[J]. Journal of Beijing Jiaotong University; 2009-04

[11] Leung D Y C, Williams D J. Modelling of Motor Vehicle Fuel Consumption and Emissions Using a Power-Based Model[J]. Environmental Monitoring \& Assessment. 2000, 65(1): 21-29.

[12] Gao Lei. Research on Vehicle Fuel Consumption Model Based on Urban Road Conditions[J]. Jilin University, 2007 